\title{
REVIEW
}

\section{Risks and Threats Comes with Global Warming: Pathogenic Free Living Amoeba}

\author{
Nihal Doğan ${ }^{1}$ \\ ${ }^{1}$ Osmangazi University Medical Faculty Microbiology Department. Eskişehir, Turkey
}

Received: 28 July 2015 accepted: 12 August 2015/ published online: 30 August 2015 (C) Ordu University Institute of Health Science, Turkey, 2015

\begin{abstract}
Free living amoebae like Naegleria, Acanthamoeba, Balamuthia and Sappinia are known appearing opportunistic and also fatal protozoa in humans and other animals. They are widely distributed in soil and water in the world. They cause "Primer Amoebic Meningoencephalitis" the host immune response to these protist pathogens differs from each other to evidence by the postmortem laboratory findings from the affected patients.

This review was performed with a search in Medline, PubMed, Science Direct, Ovid, and Scopus literatures by the search terms of "pathogenic free-living amoeba infections".

Analysis of a detailed review and literature shown that Naegleria fowleri, Acanthamoeba and Balamuthia and also Sappinia sp. infections are causing extensive brain damage to the host immune response. In human infection due to related to brain, skin, lung and eyes have increased significantly during the last years. They have different effects on epidemiology, immunology, pathology, and clinical features of the infections produced. This particular review planned to raise awareness about free-living amoeba, which found in a patient who applied to ESOGU Hospital Neurology Clinic because of suddenly unconsciousness and coma and diagnosed with Naegleria fowleri.

Clinicians should be aware of PAM infections and include in differential diagnosis of meningoencephalitis. PAM should be suspected in young adults and children with acute neurological symptoms as described below and recent exposure to fresh water. Preventive and control measures include public health education and awareness among medical practitioners and adequate control of public water supplies.

Key words: Pathogenic free-living amoebae, global warning, Naegleria, Acanthamoeba, Balamuthia, Sappinia
\end{abstract}

Address for correspondence/reprints:

Nihal Doğan

Business telephone number: +90222 23929 79/4557

E-mail: nihaldogan42@gmail.com

DOI: $10.19127 / \mathrm{mbsjohs} .94837$

\section{Introduction}

Acanthamoeba, Naegleria, Balamuthia mandrillaris and Sappinia spp. genus are members of Free-living Amoebae, (FLA), which are aerobic, eukaryotic and protists species as well. They occur worldwide and can potential cause of opportunistic infections in humans and other animals (Visvesvara et al., 2006; Visvesvara et al., 2007; Visvesvara et al., 2013). They could be found in soil, dust, air, seawater, drinking water, swimming pools, sewage, eyewash solutions, contact lens, dental treatment units, and dialysis units.

Due to the fact that these amoebae have the ability to exist as free-living organisms in nature and only occasionally invade a host and live as parasites within host tissue, they have also been called amphizoic amoeba. (Trabelsi et al., 2012; Szenasi et al., 1998). 
Taxonomy of FLA was revised several times, last data from genomic sequencing studies and based on morphologic, biochemical and molecular approaches by zoologist. This four genus FLA has been classified to two super groups; Acanthamoeba and Balamuthia classified under the super group Amoebazoa. Acanthamoebidae, N. fowleri classified under the super group Excavata: Heterolobosia. Sappinia and Vahlkampfiidae belong to super group Amoebozoa: Flabellinea: Thecamoebidae (Adl et al., 2005).

All of four amoebae are known so far to cause infections of the central nervous system (CNS). Several species of Acanthamoeba (i.e. A. castellanii, A. culbertsoni, A. hatchetti, A. polyphaga, $A$. rhysodes), the only known species of Balamuthia, B. mandrillaris, two species of Sappinia genus, S. diploidea and S. pedata, and only one species of Naegleria, N. fowleri, are known to cause disease in humans and other animals (Khan, 2006; Visvesvara et al., 2007).

A shared appearance of these species is the very large central nuclear endosome, quite different from E. histolytica, where differentiation may be necessary in tissue sections. Under dry conditions, trophozoites form became resistant cysts that permit survival and also airborne dispersal; cysts can resist chlorination. Many of species are thermophilic and are cause of "humidifier fever" and also extrinsic allergic alveolitis, fever, dyspnea, cough. Some bacteria, like Legionella and Parachlamydia, Acanthamoebae may live symbiotically within these amoebae, persisting within the phagosome, being resistant to lysosomal enzymes. Three genera of free-living amoebae cause human infections, and a few cases caused by Sappinia sp. (Trabelsi et al., 2012).

\section{Naegleria species}

Naegleria is an amoeboflagellate with two trophozoite forms. It is a thermophilic amoeba that grows well in tropical and subtropical climates. Among the 30 species of Naegleria, only one species $N$. fowleri was known human pathogen and caused a disease called Primary Amebic Meningoencephalitis (PAM). This infection characterized by an acute fulminant meningoencephalitis leading to death 3-7 days after exposure. Victims are healthy, young individuals with a history of recent water-related sport activities. This amoeba moves rapidly with a single pseudopodium, it can transform into a non-feeding flagellate in hypotonic media, and these freeswimming forms facilitate dispersal. Cysts are thin walled and spherical. The portal of entry is the olfactory neuroepithelium. The pathologic changes are an acute hemorrhagic necrotizing meningoencephalitis with modest purulent exudate, mainly at the base of the brain, brain-stem and cerebellum. Trophozoites can be seen within the CNS lesions located mainly around blood vessels. (Sugita et al., 1999; Diaz et al., 2010; Wiwanitkit 2004; Saylor et al., 2015; Lawande et al., 1980).

$N$. fowleri is thermophilic amoeba, which able to grown at $45^{\circ} \mathrm{C}$ temperature thermal water, fresh water lakes, swimming pools, but cannot survive seawater. Additionally, it was isolated from the healthy asymptomatic children nasal mucosa. Naegleria has includes amoeboid trophozoite (10$25 \mu \mathrm{m})$ and resistant cyst form $(8-20 \mu \mathrm{m})$. The trophozoite is an active form that changes size and shape; it feeds bacteria and other organic matter. Amoeboid form transforms into two flagellate forms that are usually two flagella into the distilled water, but it will convert amoeboid form when conditions are favorable. Cysts are usually spherical single walled with fine pores. Only amoeboid form is found in tissues. N. fowleri cultured non-nutrient agar plates coated bacteria and at $45^{\circ} \mathrm{C}$ like Acanthamoeba, and also axenic media (Schuster et al., 2004; Martinez, 1993).

\section{Acanthamoeba species}

Acanthamoeba is the most common amoebae and found in soil; fresh, brackish, and sea water; sewage; swimming pools; contact lens equipment; medicinal pools; dental treatment units; dialysis machines, heating, ventilating, and air conditioning systems; mammalian cell cultures; vegetables; human nostrils and throats; and human and animal brain, skin, and lung tissues. It has no flagellate form. The small pseudopodia are multiple, thin, and spike-like; they are called acanthopodia. Cysts are thick walled, angulated, and floating; their dispersal may be wind borne. Several species are pathogenic but morphological classification is unsatisfactory; rRNA sequences differentiate into 15 genotypes. Acanthamoeba is sometimes isolated from throat or nasal swabs or from stool specimens. It causes species of human infections by $A$. castellanii, A. culbertsoni, A. catchetti, A. healyi, $A$. divionensis and $A$. polyphaga. Other species are nonpathogenic and 
thermophilic (Schuster et al., 2004).

Acanthamoeba has two forms: a vegetative or trophozoite $(8-10 \mu \mathrm{m})$ and dormant cyst $(8-29 \mu \mathrm{m})$. Trophozoits has a single nucleus and a fine acanthopodia of the body. Cyst has been known to survive in vitro greater than 20 years. Laboratory cultivation is realized on non-nutrient agar medium seeded with Gram negative bacteria which are preferably not encapsulated and pigmented (Escherichia coli or Enterobacter aerogenes) to allow for growth of trophozoite forms incubated $30^{\circ} \mathrm{C}$. They can also axenic medium and mammalian cell cultures.

Acanthamoeba can cause Granulomatous amoebic encephalitis (GAE) cutaneous and nasopharyngeal infections in immunocompromised patients a painful keratitis generally in contact-lens users. Acanthamoeba infections are rarely encountered in humans and animals, but showed that Acanthamoeba antibodies more than $80 \%$ of normal human populations (Chappell et al., 2001). Cutaneous acanthamoebiasis is cause rarely opportunistic infections in immunocompromised patients, especially HIV infected. Characteristic lesions are nodules, fistulas, papules and skin ulcerations contains with cyst and trophozoits. Lesions are usually face, trunk and extremities and spread blood stream of other tissues (Visvesvera et al., 2007; Patel et al., 2010).

Acanthamoeba keratitis (AK) is usually developed immunocompetent persons a corneal infection. The main risk factors are use of extended contact lens trauma. The lesion is typically only one eye involved. In recently, AK cases have been increasing in developing countries - correlating to contact lens wearers (85\%) (Visvesvera et al., 2007; Patel et al., 2010; Tu et al., 2010).

\section{Balamuthia mandrillaris}

Balamuthia is closely related to Acanthamoeba and not a leptomyxid amoeba; it shows little directional movement and has an irregular or branched shape. Cysts are thick walled and spherical. Human infections formerly attributed to Hartmannella sp. are now all thought to be due to Balamuthia mandrillaris, a species described in 1993 from a mandrill baboon that died of meningoencephalitis in San Diego zoo. Balamuthia can only be cultured on tissue culture monolayers. About 100 cases have been reported worldwide, but many are from Latin America. B. mandrillaris like several species of Acanthamoeba are pathogenic "opportunistic" free-living amoebas which cause Granulomatous Amebic Encephalitis (GAE) in humans and animals. GAE is an infection, usually seen in debilitated, malnourished individuals, in patients undergoing immunosuppressive therapy for organ transplants and in AIDS. The granulomatous component is negligible, particularly in immunocompromised individuals. Pathologically these amoebas produce patchy, chronic or sub-acute granulomatous encephalitis with the presence of trophozoites and cysts. The portal of entry is probably through the respiratory tract or an ulceration of the skin reaching the CNS by hematogenous spread. $B$. mandrillaris does not feed on bacteria and cultured non-nutrient agar. These amoeba growth in also tissue cultures. Its life cycle comprises trophic amoeboid stage $(50-60 \mu \mathrm{m})$ and cyst $(12-30 \mu \mathrm{m})$ with triple layer wall no pores. They may be found in brain, eyes, skin, lungs and other organs (Visvesvara et al., 2007; Saylor et al., 2015).

\section{Sappinia species}

Sappinia genus have comprises of $S$. pedata and $S$. diploidea name of two distinct species. They widely found of mammalian feces, soil, freshwater, forest liter, elk, bison, cattle and lizard rectum. It has two form trophozoit (40-80 $\mu \mathrm{m}$ and cyst $18-25$ $\mu \mathrm{m})$. Cysts are double walled and have nuclear pores and it can survive passage through the stomach. Sappinia $s p$. has not been shown to be lethal in human and experimental animals and never implicated pathology. Only one reported of encephalitis immunocompetant young man who survived. This cases caused by Sappinia may developed from earlier sinus infection and developed unconscious, seizure and also solitary mason sectioning showed necrotizing hemorrhagic inflammation of containing trophozoite form especially blood vessels. The patient's outcome after surgical excision of necrotic lesion, and successful treated of azitromycin, pentamidine, Itroconazole. A more phylogenetic study is needed to indicated that species this genus (Gelman et al., 2003; Visvesvera et al., 2007).

\section{Free-living amoebae; Epidemiology, Pathology and Clinical Finding}

FLA infected patients are usually give a history of swimming or diving in warm fresh water or spa water between 2 and 14 days before the illness began. Common-source outbreaks occur during warm summer months in temperate countries. This waterborne disease was discovered in Australia in 
the 1965. Since then, it has been reported from about 15 other countries in Africa, Asia, Europe and North and South America (Heggie, 2010; Khan, 2006; Szenasi et al., 1998; Trabelsi et al., 2012; Visvesvera et al., 2006).

\section{Primer Amoebic Meningoencephalitis (PAM)}

PAM is caused by Naegleria fowleri, it fallows intranasal infection during swimming in warm, contaminated freshwater. Most victims have been children or young adults and the disease is almost invariably fatal (WHO Library "Emerging issues in water and infectious diseases series, Visvesvara et al., 2007; Heggie, 2010). Recent studies shown that in the USA were identified, Australia was the only country where $N$. fowleri has been associated with public water supplies. In USA recently, the causal agent of a PAM case in the US was diagnosed as Paravahlkampfia francinae a new species of the free-living amoeba genus Paravahlkampfia that was isolated from the cerebrospinal fluid of a patient with headache, sore throat, and vomiting, presenting typical symptoms of PAM caused by $N$. fowleri. Thus awareness of novel emerging amoebae as causative agents of PAM should also be considered (Visvesvara et al., 2009; Kristenssons 2013). Amoebic trophozoites pass the cribriform plate from the nasal mucosa to the olfactory bulbs and subarachnoid space. In postmortem examination of the brain shows cerebral softening and damage to the olfactory bulbs; cysts are never formed in the tissues. The earliest symptoms are sudden headaches, high temperature, and nuchal rigidity, followed by nausea, vomiting, irritability and restlessness. Nuchal rigidity usually occurs with positive Kernig and Brudzinski signs. Photophobia may occur late in the clinical course, followed by neurological abnormalities, including lethargy, seizures, confusion, coma, diplopia or bizarre behavior, leading to death within a week. Cranial nerve palsies (third, fourth, and sixth cranial nerves) may indicate brain edema and herniation. Intracranial pressure is usually raised to $600 \mathrm{~mm}_{2} \mathrm{O}$ or higher. Cardiac rhythm abnormalities and myocardial necrosis have been found in some cases. Death is usually increased intracranial pressure with brain herniation, leading to cardiopulmonary arrest and pulmonary edema. Rarely, chronic and progressive infection of the CNS may involve the lungs (da Rocha-Azevedo et al., 2009). AGE is usually associated with an underlying debilitating disease or immune suppressed individuals including HIVAIDS patients and diabetics. More than 200 cases have been documented since the first human case was reported in 1995. But, some of them are missed clinically and discovered at autopsy or in preserved pathological material. Specific antisera enables amoebae to be recognized by immunofluorescence staining (Martinez et al., 1980; Visvesvara et al., 2006; Visvesvara et al., 2007; Khan, 2006; da Rocha-Azevedo et al., 2009). Immunocompetent patients are usually young adults and children. At first, nasal symptoms and headache are soon followed by fever, neck rigidity, coma, and, later, convulsions; mostly it is fatal within a few days. CSF is usually turbid and bloody with high protein, low glucose and neutrophils. Amoebae must be urgently looked for wet specimens using phase-contrast microscopy. Unless amoebae are seen, bacterial meningitis will be suspected; on Gram staining amoebae appear as indistinct smudges. Fixed preparations stained with iron haematoxylin will show full details of nuclear structure. Confirmation is by culture at $37^{\circ} \mathrm{C}$ using a gram negative bacterial lawn on non-nutrient agar (Visvesvara et al., 2007).

\section{Amoebic Keratitis (AK)}

Infections are usually due to Acanthamoeba sp. and also associated with contact lens users. Risk factors include poor hygiene when handling lenses and their cases, use of chlorine-based disinfectants, swimming or washing eyes while wearing lenses, after gardening and too prolonged use of plastic or unwashed lenses. Corneal lesions are painful and present as painless and progressive ulcers leading eventually to perforation. Frequently confused with microbial keratitis, including Pseudomonas, Staphylococcus and Herpes simplex. Amoebae are found in corneal scrapings or histologically in corneal tissue, but can be missed unless stained with iron haematoxylin or immunofluorescence. PCR is an available method now. Fresh material is using for cultures from a bacterial lawn on nonnutrient agar, should be at $30^{\circ} \mathrm{C}$. They usually (90\%) of cases are due to genotype T4 (Khan, 2006; Visvesvara et al., 2007; Martín-Navarro et al., 2008; da RochaAzevedo et al., 2009; Verani et al., 2009; Tu and Joslin, 2010; Patel et al., 2010). 


\section{Granulomatous Amoebic Encephalitis (GAE)}

Infection causes due to Acanthamoeba and Balamuthia and the main route of the lower respiratory tract followed by haematogenous spread to the brain. Other routes of entry are the skin, the nasopharynx, the lungs and the stomach. Primary lesions have been described at all these sites. Many species of Acanthamoeba can cause GAE, also known as Acanthamoeba Granulomatous Encephalitis (AGE). AGE is a rare, chronic, progressive infection of the CNS that may involve the lungs (da Rocha-Azevedo et al., 2009). That is usually associated with an underlying debilitating disease or immune suppressed individuals including HIV-AIDS patients, diabetics (Khan, 2006; Visvesvara et al., 2007; da RochaAzevedo et al., 2009).

B. mandrillaris are soil contamination of skin and craniofacial wounds is an important risk factor. Causes of immunocompromise include malignancy, collagen disorder, alcoholism, diabetes mellitus, AIDS, and steroid or immunosuppressant therapy. Recently two patients with $B$. mandillaris infection have been described both of whom had received a kidney graft from the same donor. However, in

Peru, most of the patients infected with $B$. mandrillaris have no obvious cause for immunosuppression. Amoebic lesions resemble chronic bacterial brain abscesses or localized subacute hemorrhagic necrosis; involvement of the meninges is common and also present with headache and meningism, others with evidence of a focal brain lesion. Unless these amoebae are found in wet tissue preparations or cerebrospinal fluid, the diagnosis will be usually based on histology, often at autopsy. Cysts may be seen in tissue but trophozoites may be missed unless stained with iron haematoxylin or immunofluorescence using specific antisera. Cultural diagnosis at $37^{\circ} \mathrm{C}$ from fresh biopsies or cerebrospinal fluid is sometimes possible. PCR methods are becoming available (Deetz et al., 2003; Schuster et al., 2006).

In addition to the number of affected individuals is increasing worldwide. Moreover, both Acanthamoeba and Balamuthia cause infections of the lungs and skin (Khan, 2006; Maciver, 2007; Visvesvara et al., 2007; da RochaAzevedo et al., 2009). Recently, B. mandrillaris, has been discovered to cause a fatal encephalitis in humans (Martin et al., 2014). This encephalitis is known as Balamuthia amoebic encephalitis (BAE). There are worrying features of BAE that are emerging, even compared to AGE and PAM. PAM is restricted to bodies of warm freshwater, such as swimming pools and lakes, and so can be avoided after its presence has been identified. AGE is mostly a disease of the immunocompromised, and so affects a small subpopulation of individuals who could conceivably be monitored for early signs of AGE; for example, by inspection of cerebrospinal fluid (CSF) (Deetz et al., 2003; Maciver, 2007). The unpredictable nature of the disease may means that $\mathrm{BAE}$ is even less likely to be diagnosed in time for medical intervention and, like AGE and PAM, it is essential for BAE to be diagnosed early if it is to be treated successfully (Deetz et al., 2003; Maciver, 2007). Additionally; Sappinia diploidea and Sappinia pedata species, also belonging to the free-living amoeba group, that normally live in soil contaminated with feces of elk, bison, and cattle, have been identified as causing encephalitis in an otherwise healthy individuals (Gelman et al., 2001; Qvarstrom et al., 2009; Walochnik et al., 2009). In a retrospective study shown that, there are at least 13.939 cases of acute encephalitis diagnosed between 1990 and 1999 in California, among these cases, $0.1 \%$ was attributed to Naegleria, $0.63 \%$ to other protozoans, and $34.7 \%$ were from unspecified causes. (Maciver et al., 2007)

\section{Treatment of Free Living Amoeba}

Therapy and prognosis of FLA infections is problematic because of the lack of clear-cut symptoms, the lack of a good reliable diagnostic test, and the fact that diagnosis is often made postmortem. However, several patients with GAE caused by Acanthamoeba spp. as well as some with Acanthamoeba cutaneous infection without CNS involvement have been successfully treated with a combination of pentamidine isethionate, sulfadiazine, flucytosine, and fluconazole or itraconazole. Topical applications of chlorhexidine gluconate and ketoconazole cream in addition to antimicrobials have resulted in therapeutic success for cutaneous Acanthamoeba infection, but in many cases, had to be discontinued because of undesirable side effects of the medications (Visvesvara et al., 2007).

A combination of factors of late diagnosis, suboptimal efficacy of antimicrobial therapy, and problems inherent to the immunocompromised host, makes for a poor prognosis for GAE patients. Treatment of Acanthamoeba keratitis has been 
fairly successful. A variety of drugs have been used, including chlorhexidine, polyhexamethylene biguanide, propamidine isethionate, dibromopropamidine isethionate, neomycin, paromomycin, polymyxin B, clotrimazole, ketoconazole, miconazole, and itraconazole (Visvesvara et al., 2007; da RochaAzevedo., 2009).

A few patients have survived to $N$. fowleri caused by PAM. Lately used of aggressively treated with intravenous and intrathecal Amphotericin B, intravenous and miconazole, also oral rifampin. It was believed that Amphotericin B and miconazole had a synergistic effect but that rifampin was without effect on the amoebae. Based on in vitro testing and in vivo mouse studies, amphotericin $\mathrm{B}$ was reported to be more effective against $N$. fowleri than amphotericin B methyl ester, a water-soluble form of the drug (Visvesvara et al., 2007; da Rocha-Azevedo et al., 2009). Additionally, Azithromycin

has been shown to be effective a macrolide antimicrobial against both in vitro and in vivo and also sensitive to voriconazole. However; other macrolides (erythromycin, clarithromycin) are less effective this parasite (Visvesvara et al., 2007; da Rocha-Azevedo et al., 2009). Another drug, miltefosine, an alkylphospholipid, has also been shown to have amoebicidal potential. Significantly, medical cure has been achieved with the application of either polyhexamethylene biguanide (PHMB) or chlorhexidine gluconate with or without Brolene. When medical treatment failed, a combination of debridement and penetrating keratoplasty has been used with good results in some cases (Schuster et al., 2006).

Balamuthia amoebic encephalitis (BAE) is a rare, sub-acute to chronic disease that is characterized by hemorrhagic necrotizing lesions or brain abscess (Bravo and Sanchez, 2003). They have been treated empirically with steroids as well as with antibacterial, antifungal and antiviral agents with almost no effect upon the course of the infection. Anti-inflammatory steroids that were administered may have actually facilitated spread of the infection by suppressing the inflammatory response (Deetz et al., 2003; Martínez et al., 2010; Bravo et al., 2011).

In vitro studies have shown that pentamidine and propamidine isethionates were amoebastatic but not amoebicidal. Recent information based on in vitro data has shown that miltefosine was able to lyse the amoebae. Voriconazole, however, had virtually no effect on Balamuthia (Maciver., 2007; Visvesvara et al., 2007).

Recently, novel therapeutic approaches of the application of siRNA in Acanthamoeba species (Lorenzo-Morales et al., 2005; 2008; 2010) has opened a novel approach for the progress of future therapies based on siRNAs alone or in combination with chemical compounds.

\section{Conclusion}

FLA infections are emerging diseases that gained recently much attention. They are ubiquitous and widely organisms in the worldwide. In the future they will be increasingly dangerous because of thermophilic and polymorphic structure, easy adaptation and resistible cyst wall. In patients who have meningitis symptoms, FLA is an important agent to be considered. In conclusion, there is a vital necessity of simple and rapid methods of diagnosis for these fatal amoebae that live in fresh water sources.

Peer-review: Externally peer-reviewed.

Author Contributions: Concept ND; Design ND; Supervision ND; Data Collection and/or Processing $\mathrm{ND}$; Analysis and/or Interpretation ND; Literature Review - ND; Writing ND; Critical Review ND.

Conflict of Interest: No conflict of interest was declared by the authors.

Financial Disclosure: The authors declared that this study hasn't received any financial support.

\section{References}

Adl SM, Simpson AGB, Farmer MA, Andersen RA, Barta JR, Bowser SS, et al. The new higher level classification of eukaryotes with emphasis on taxonomy of protists. J Eukaryot Microbiol 2005; 52: 399-451.

Bravo FG, Seas C. Balamuthia mandrillaris amoebic encephalitis: an emerging parasitic infection. Curr Infect Dis Rep 2012; 14(4):3916.

Chappell CL, Wright JA, Coletta M, Newsome AL. Standardized method of measuring Acanthamoeba in sera from healthy human subjects. Clin Diagn Lab Immunol 2001; 8:72430

da Rocha-Azevedo B, Tanowitz HB, MarcianoCabral F. Diagnosis of infections caused by pathogenic free-living amoebae. Interdiscip Perspect Infect Dis 2009: 2009; 251406. 
Deetz TR, Sawyer MH, Billman G, Schuster FL, Visvesvara GS. Successful treatment of Balamuthia amoebic encephalitis: presentation of 2 cases. Clin Infect Dis 2003; 15;37(10):1304-12.

Diaz J. Seasonal primary amebic meningoencephalitis (PAM) in the south: summertime is PAM time. J La State Med Soc. 2012; 164(3):148-150.

Free Living Amebic infections. Images library, page 5. Available from:URL: http://dpd.cdc.gov/dpdx/HTML/Image library/AF/Free living amebic/body,htm.

Gelman BB, Popov V, Chaljub G, nader R, rauf SJ Nauta HW: Neuropathological and ultrastructural features of amobic encephalitiscaused by Sappinia diplidea. J Neuropathol Exp Neurol 2003:62: 990-8.

Heggie TW, Swimming with death: Naegleria fowleri infections in recreational waters. Travel Med Infect Dis. 2010; 8(4):201-6.

Khan N.A. Acanthamoeba: biology and increasing importance in human health, FEMS Microbiol Rev, 2006; 30: 564-595.

Kristensson K, Masocha W, Bentivoglio M. Mechanisms of CNS invasion and damage by parasites. Handb Clin Neurol 2013;114:11-22.

Lawande RV, Macfarlane JT, Weir WR, AwunorRenner C. A case of primary amebic meningoencephalitis in a Nigerian farmer. Am J Trop Med Hyg 1980; 29(1): 21-5.

Maciver SK. The threat from Balamuthia mandrillaris. J Med Microbiol 2007; 56:1-3.

Martinez AJ. Free-living amoebas: infection of the central nervous system. MT Sinai J Med 1993; 60(4):271-8.

Martín-Navarro CM, Lorenzo-Morales J, LópezArencibia A, Reyes-Batlle M, Piñero JE, Valladares B, Maciver SK. Evaluation of Acanthamoeba myosin-IC as a potential therapeutic target. Antimicrob Agents Chemother 2014; 58(4): 2150-5.

Marciano-Cabral F. Advances in free-living amebae research 2006: summary from the IX International Workshop on Opportunistic Protists (IWOP-9). J Eukaryot Microbiol 2006; 53 Suppl 1:S8-9.

Patel DV, Rayne S, McGhee CN Resurgence of Acanthamoeba keratitis in Auckland, New Zealand: a 7-year review of presentation and outcomes. Clin Experiment Ophthalmol. 2010; 38(1):15-20
Qvarnstrom Y, da Silva AJ, Schuster FL, Gelman $\mathrm{BB}$, Visvesvara GS.Molecular confirmation of Sappinia pedata as a causative agent of amoebic encephalitis. J Infect Dis 2009; 15;199(8):1139-42.

Saylor D, Thakur K, Venkatesan A. Acute encephalitis in the immunocompromised individual. Curr Opin Infect Dis 2015; 28(4):330-6.

SchusterFL, Guglielmo BJ, Visvesvara GS. Invitro activity of miltefosine and voriconazole on clinical isolates of free-living amebas: Balamuthia mandrillaris, Acanthamoeba spp., and Naegleria fowleri.,J Eukaryot Microbiol, 2006;53(2):121-6.

Schuster FL. Cultivation of pathogenic and opportunistic free-living amoeba. Clin Microbiol Rev 2002: 15: 342-54.

Schuster FL, Visversvara GS. Free-living amoebae as opportunistic and nonopportunistic pathogens of human and animals. Int J Parasitol 2004; 34: 1001-27.

Sugita Y, Fujii T, Hayashi I, Aoki T, Yokoyama T, Morimatsu M, Fukuma T, Takamiya Y. Primary amebic meningoencephalitis due to Naegleria fowleri: an autopsy case in Japan, Pathol Int 1999; 49(5): 468-70.

Szenasi Z, Endo T, Yagita K, Nagy E, Isolation, identification and Increasing importance of Free living amoebae causing human diseases.J Med Microbiol 1998; (47): 5-16.

Trabelsi H, Dendana F, Sellami A, Cheikhrouhou F, Neji S, Makni F, Ayadi A: Pathogenic freeliving amoebae: Epidemiology and clinical review. Pathologie Biologie, 2012; 60; 399405.

Tu EY, Joslin CE, Shoff ME. Successful treatment of chronic stromal Acanthamoeba keratitis with oral voriconazole monotherapy. Cornea 2010; 29(9): 1066-8.

Verani JR, Lorick SA, Yoder JS, Beach MJ, Braden CR, Roberts JM, Conover CS, Chen S, McConnell KA, Chang DC, Park BJ, Jones DB, Visvesvara GS, Roy SL National outbreak of Acanthamoeba keratitis associated with use of a contact lens solution, United States. Emerg Infect Dis 2009 ; 15(8):1236-42.

Visvesvara GS. Infections with free-living amebae Clin Neurol 2013; 114:153-68. 
Visvesvara GS, Maguire J.H. Pathogenic and opportunistic free-living amoebae: Acanthamoeba spp, Balamuthia mandrillaris, Naegleria fowleri, and Sappinia diploidea Trop Infect Dis 2006; 1114-1125.

Visvesvara, G.S, Moura H, Shuster FL.Pathogenic and opportunistic free-living amoebae: Acanthamoeba spp., Balamuthia mandrillaris, Naegleria fowleri and Sappinia diploidea, FEMS Immunol Med Microbiol 2007; 50: 1-26.

Walochnik J, Wylezich C, Michel R. The genus Sappinia: history, phylogeny and medical relevance. Exp Parasitol 2010; 126(1):4-13.

Wiwanitkit V, Review of clinical presentations in Thai patients with primary amoebic meningoencephalitis. MedGenMed 2004; 8: 6(1):2.

WHO Library Cataloguing-in-Publication Data Pond, Kathy. Water recreation and disease. Plausibility of Associated Infections: Acute Effects, Sequelae and Mortality / K. Pond (Emerging issues in water and infectious diseases series. 